\title{
Expertise practice of clinical transplant coordinators in Japan
}

\author{
Kuniko Hagiwara*1, Natsuko Seto ${ }^{2}$, Yasuko Shimizu ${ }^{3}$ \\ ${ }^{1}$ Division of Nursing, Department of Transplantation Medicine, Osaka University Hospital, Suita, Osaka, Japan \\ ${ }^{2}$ Faculty of Nursing, Kansai Medical University, Hirakata, Osaka, Japan \\ ${ }^{3}$ Division of Health Sciences, Graduate School of Medicine, Osaka University, Suita, Osaka, Japan
}

Received: September 29, 2017

DOI: $10.5430 /$ jnep.v8n6p99
Accepted: January 4, 2018

URL: https://doi.org/10.5430/jnep.v8n6p99

\begin{abstract}
Objective: This study aimed to describe the expertise practice of nurse clinical transplant coordinators (CTCs) in organ transplantation medical care.

Methods: To present the expertise practices of CTCs, we conducted a qualitative descriptive study using semistructured interviews. We performed an analysis according to analytical methods from the (Modified) Grounded Theory Approach (M-GTA) proposed by Kinoshita.

Results: The expertise practice of CTCs was associated with 14 categories: [conscious communication toward compromise], [observation to understand actual intention], [conversation content that quickly notices the other's reaction and changes], [engaging so that patients and families do not have regrets after a transplantation], [provide support for the self-determination process and advocate the decision], [autonomous judgment and timely response while assessing patient conditions], [coordination in response to changes in conditions], [assertive communication with physicians], [care that is aware of team medicine], [mediate to build trust between patients/families and medical staff], [long-term care as a permanent primary], [promoting self-monitoring by grasping the timing of guidance], [stepping forward to solve problems], and [evaluate their own ability appropriately and have humble attitude].

Conclusions: CTC in Japan demonstrates expertise practice with decision support during transplant selection, coordination during the transplant process, and long-term continuing care. In addition, this study highlighted that CTCs in Japan had been conducting autonomous practice as a nurse and providing comprehensive care including patients, family members, and living donors. Our results clarified the experienced CTC's expertise practices in all organ transplants and can be used to improve and assess the quality of care given by Japanese CTCs in the transplant process.
\end{abstract}

Key Words: Transplantation, Transplant coordinator, Expertise practice, Nursing practice

\section{INTRODUCTION}

Organ transplantation was developed in Western countries. In the United States, 3,606 organ transplants were performed in $2016,18 \%$ of which were living organ transplants. ${ }^{[1]}$ In Japan, 338 deceased donor transplantation ${ }^{[2]}$ and 1,852 living donor kidney and liver transplants have been performed in $2016 .^{[3,4]}$

Historically, transplant coordinators have been involved in the entire transplant continuum, from donor identification and organ retrieval to long term postoperative management

\footnotetext{
* Correspondence: Kuniko Hagiwara; Email: hagiwara@hp-trpmed.med.osaka-u.ac.jp; Address: Division of Nursing, Department of Transplantation Medicine, Osaka University Hospital, Suita, Osaka, Japan.
} 
of the recipient. Over the years, as technology became more complex and transplant volume increased, the transplant coordinator's responsibilities expanded. Eventually, this single evolved into two roles for care of organ donor and care of recipient. ${ }^{[5]}$ Currently in the US, transplant coordinators who oversee organ donation are termed the procurement transplant coordinator, whereas those in charge of transplantation are called the clinical transplant coordinator (CTC), both of which are established independent occupations. CTCs consist of nurses (e.g., critical care nurses, physician assistants, nurse practitioners, and dialysis nurses) and clinicians trained at the doctorate level. ${ }^{[6]}$ In Japan, the term CTC denotes the recipient transplant coordinator (RTC). In a survey conducted by the Japan Transplant Coordinator Organization in 1999, RTCs were established in 21 of the 102 facilities that responded to a survey conducted on 188 transplantation facilities. Of these 21 facilities, $83 \%$ were medically certified and only two dedicated RTCs were nurses. ${ }^{[7]}$ However, the results of the 2008 survey revealed the existence of 58 RTCs, $97 \%$ of whom were medically certified and $85 \%$ were nurses (51); the ratio of nurses exhibited a significant increase. ${ }^{[8]}$

For several years, CTCs in the US have been functioning at an advanced practice level, and their role is defined with specialized expertise achieved over time. ${ }^{[6]}$ CTCs are key members in a multidisciplinary team. CTCs are responsible for patient evaluation before transplantation, as well as making all arrangements during the wait period and following transplantation. ${ }^{[9]}$ RTCs have four major roles in Japan: (1) evaluation and assistance with psychological and social problems, and providing support to patients and their families regarding decisions related to transplantation; (2) facilitating communication and coordination within the transplantation team throughout the course of treatment; (3) providing continuous guidance and education to patients and their families before and following surgery; and (4) conducting long-term medical follow-up of patients and living donors. ${ }^{[10]}$ The certification system to become RTC was established in 2011. Certified RTCs are responsible for adjusting the transplantation team, advising patients on activities of daily living before and after the transplantation as well as providing physical management and mental care throughout the transplantation process. ${ }^{[10]}$

The reports on the roles and tasks of RTC nurses and practical reports are limited to specific organ transplants and operations. ${ }^{[11-17]}$ However, the RTC being a nurse is essential to fulfill all such roles, and, from the viewpoint of nursing practice, the overall perspective of RTC nurse (CTC) practice has not yet been clarified. We clarified the aspect of expertise practice of RTC nurses in Japan, developed in the transplant process common to all organs. With regard to the abilities of Japanese CTCs, the results of a comparison of Japanese nursing education curriculum with NATCO's core competencies revealed outstanding practice, education, consultation, adjustments, and ethics, which are equivalent to the traits desired in a certified nurse specialist (CNS). ${ }^{[18]}$ In the US, CTCs have been practicing at a high level with specialized, complex skills and knowledge for at least 30 years. ${ }^{[6]}$ Advanced practice nurses (APNs) and registered nurse respondents and are separate from the duties of the CTC in the United States. ${ }^{[19]}$ In addition, within the transplant team, CTCs, APNs, such as nurse practitioners, and clinical nurse specialists carry out multiple nursing roles to ensure safe care of patients, families, and donors on the basis of their individual required licensing state-mandated scope of practice. ${ }^{[20]}$ However, in Japan, the educational system and definition of nursing practice abilities necessary for CTCs have yet to be established, and actual details depend on individual experience and recognition. Although the history of the CTC in Japan is shorter than that in the United States, we thought that CTCs in Japan were becoming expert by experience. It is important to clarify that the experienced CTC's expertise practice also leads to assessment of the quality of nursing practice at the CTC level. Also, to describe universal activities as CTC's expertise practices, we thought it necessary to find common practices in treatment of all organs. We hypothesized that understanding the practice of well-experienced Japanese RTCs would elucidate the expertise practice of CTCs in Japan. Hence, this study aimed to describe the expertise practice of Japanese nurse CTCs in all organ transplantations.

\section{Methods}

\subsection{Study design}

To present the expertise practice of CTCs, we conducted a qualitative descriptive study using semistructured interviews.

\subsection{Operational definition of terms}

CTC: In 2004, the Japanese Nursing Association defined CTCs as nurses who are highly skilled with cutting-edge and diverse expert knowledge on transplantation, and autonomously take on the role to make the required adjustments to ensure that subjects of the entire transplantation process can receive the best transplant medical care. In this study, we define a nurse named recipient transplant coordinator in Japan as a clinical transplant coordinator.

\subsection{Study subjects}

Certified recipient transplant coordinator nurses who are active in Japan, have $\geq 5$ years of experience as RTCs, and are a dedicated/full-time RTCs. 


\subsection{Data collection methods}

We mailed or handed the request for the research objective in person to certified recipient transplant coordinators who were certified by the first Joint Committee for Certification of Recipient Transplant Coordinators. Among CTCs who provided consent, we enrolled those with $\geq 5$ years of experience of being CTC and with expertise in all organ transplantation to obtain variable data for 11 subjects. The interview survey was conducted from January 2013 to December 2014, and for each period (before transplantation, from operation to discharge, and after discharge), the care they provide as CTCs was examined as open-ended responses to questions using a semistructured interview. With permission, we recorded the interview with an IC recorder. Details of the interview were later recorded as a transcript.

\subsection{Analytical methods}

We used the modified grounded theory approach (MGTA), ${ }^{[21,22]}$ which is a qualitative analysis method derived from the original grounded theory approach, for the analysis. In this study, the analytic them was "What do CTC demonstrated the practice in the transplant process?" The focused person for analysis was "CTC who are nurse". We first gained a comprehensive understanding of the interview data and then analysed as follows: (i) We useed an analysis worksheet with four columns: examples, theoretical notes, concept definition, and concept name. We used one worksheet to generate one concept. First we selected interview related to the analytic theme recorded them in the example column; (ii) We used comparative analysis to examine other similar/contrary examples; (iii) We aimed to find a common meaning among the many examples; for coding, we described the concept difinition and the concept name in a short statement; (iv) Once we generated multiple concepts, we described the concepts, we initiated the process to examine the interconcept relationship to clarify their directionality and generate a category under which these concept fell; (v) We developed a schematized diagram of the relationshop between concepts and categories; (vi) We judged that theoretical saturation had been reached when no additional concepts could be generated and when available concepts and categories were deemd sufficient to explain the phenomena described by the analytic theme. This research was supervised by two researchers specialized in qualitative nursing studies.

\subsection{Ethical considerations}

This study was approved by the Medical Ethics Committee of Osaka University . Before the interviews, all participants were explained about the purpose of this study, research methods, length of interviews, use of results, identity of re- searchers, anonymity of participants, and potential risks and benefits of research participation. All participants signed a consent form after being informed that they could quit at any time during the interviews. Times that were convenient for participants were selected for the interviews, which were conducted in a private room to ensure confidentiality. The interviews were recorded and then transcribed. The recorded tapes were deleted, and the transcript was stored in a locked cabinet by the researcher responsible for data.

\section{ReSUltS}

\subsection{Summary of subjects}

The subjects consisted of 11 certified recipient transplant coordinator nurses (CTCs) who provided consent. Of these, eight nurses were full-time nurses, three worked exclusively in transplant care, and all were female. The mean duration of experience as a nurse was 21 years (13-26 years), the mean duration of experience as CTCs was 10 years (5-17 years), six CTCs were in charge of transplants of one organ, and five were in charge of transplants of multiple organs. The organ breakdown was as follows (multiple answers): three heart transplants, one lung transplant, five liver transplants, two pancreas transplants, four kidney transplants, and two small intestine transplants.

\subsection{Storyline of CTC expertise practice}

Regarding decision-making support provided by CTCs in transplant decisions, the problem was captured from the thoughts and relationship of family members through [observation to understand actual intention]. In an interview, a comfortable environment was created through [conscious communication toward compromise] to ensure that the individual could speak candidly. Through [communication with a flow in response to the other person's reaction], provision of appropriate information on transplantation and the level to which the content was understood was attempted in the conversation. The use of these practices [engaging so that patients and families do not have regrets after a transplantation] led to decision making that included family members. As CTCs, they [provide support for the self-determination process and advocate the decision] of patients and living donor candidates with an ethical approach.

Throughout the transplantation process, CTCs implemented [autonomous judgment and timely response while assessing patient conditions,] according to medical knowledge, toward treatment by noticing patient responses; determining transplant suitability and patient abnormalities; making adjustments to sudden problems that occur during the transplantation process; and practicing [coordination in response to changes in conditions] using any method possible. To 
practice the best medical care to patients, CTCs attempt to provide [collaborative care that is aware of team medical care] that is available and conduct [assertive communication with physicians]. Furthermore, they actively [mediate to build trust between patients/families and medical staff].

In the guidance of post-discharge daily life, they promote post-transplantation awareness of health management to patients through [promoting self-monitoring by grasping the timing of the guidance], and providing post-transplantation daily life support to patients and family through [long-term care as a permanent primary].

In all practices, CTCs have an attitude of [stepping forward to solve problems], and aim to achieve better coordination by carefully considered problem solving. CTCs reflect on their activities each day, and expand their practices according to their experiences to appropriately [evaluate their own ability and maintain a humble attitude]. This has led to their own growth (see Figure 1).

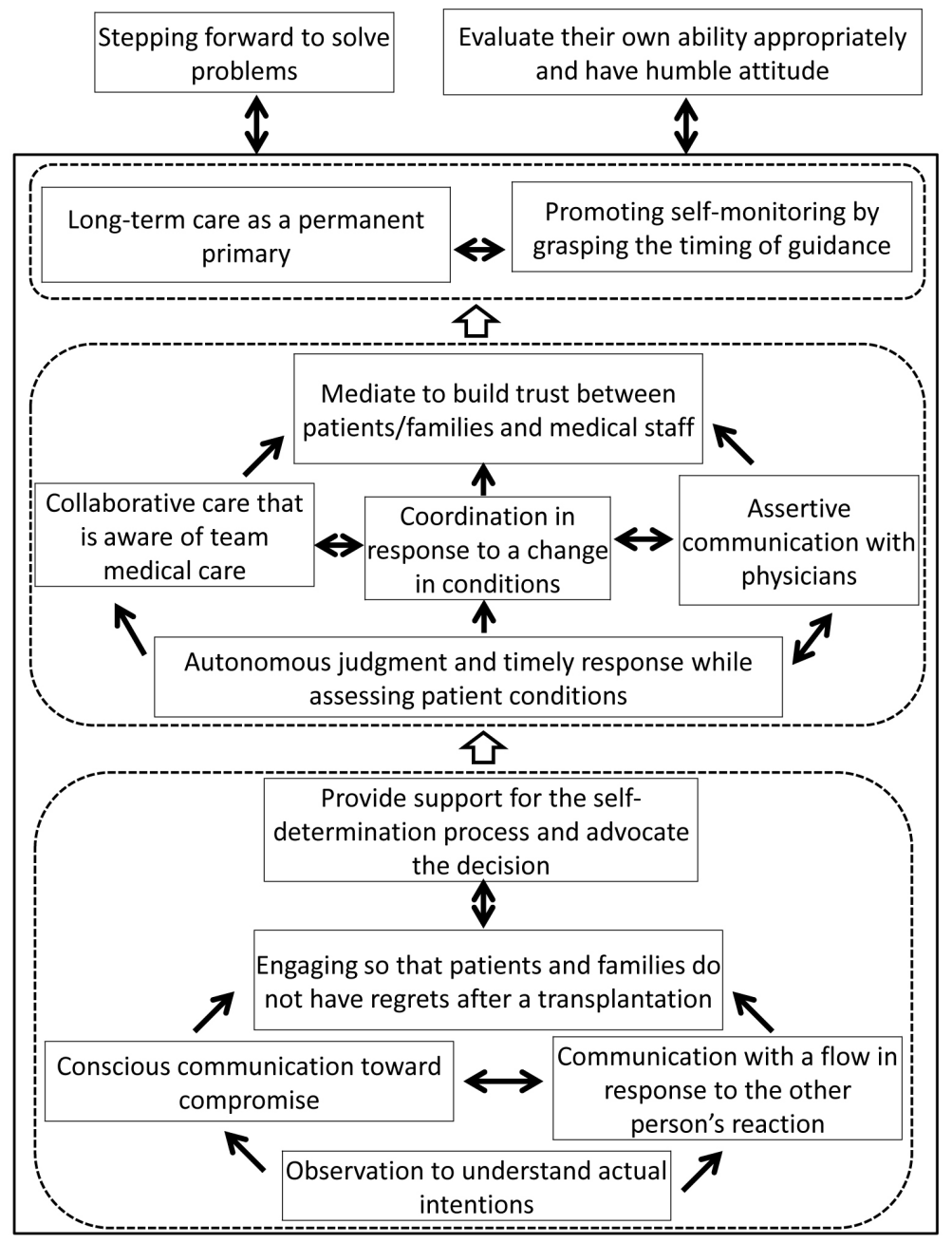

Figure 1. Expertise practice of clinical transplant coordinators in Japan

3.3 Categories and concepts that constitute expertise practices

For expertise practices of CTCs, we generated 14 categories and 49 concepts. We present categories with [ ], concepts with .

\subsection{1 [Observation to understand actual intentions]}

The category of [observation to understand the real intention] consisted of three concepts: careful observation to examine readiness for transplantation, understanding hesitations living donor candidates have about donation, and capture problems based on facial expressions and the words of living donor candidates and families.

[Observation to understand actual intention] was a form of expertise practice that became useful during decision-making support in transplantation decisions. During the first contact with patients and families (e.g., an initial explanation of transplantation), CTCs make careful observations to examine readiness for transplantation with critical eyes to determine 
whether the recipient and donor truly desire the transplantation, and intervene to determine the true intention. It is important for CTCs to understand hesitations living donor candidates have about the donation in a family relationship in which it appears unlikely that the donor volunteered, or for donor candidates who are in a socially weak position, and create a way out so that their donation can be turned down. They capture problems based on facial expressions and the words of the living donor candidates and family members to understand the true intention.

One CTC described:

As a coordinator, depending on the expression of family members and patients, how to nod to the teacher at the time of questioning, and the contents of questions from patients; some of these people probably do not have families who do not really want to transplant. I try to listen to the story while feeling that the patient is brought in such a way that she is "pumped by my family" and is moving toward the transplant. ( $\mathrm{J}$ )

\subsection{2 [Conscious communication toward compromise]}

The category of [conscious communication toward compromise] consisted of three concepts: conscious effort to make an open atmosphere for free discussion, attitude to wait until the patients are ready to talk, and actively compromise.

[Conscious communication toward compromise] is the ability to communicate achieved through the need to discover the relationship and problems associated with patients and families during the first interview. CTCs make a conscious effort to make an open atmosphere for free discussion in which patients feel comfortable to discuss private issues and true intentions. Communication with patients to elicit their true intentions involves an attitude of waiting until the patients are ready to talk, and not only asking what they want to hear but also waiting patiently even if patients are quiet. Even with patients they are not comfortable with, they actively compromise and engage with an open heart. In this manner, CTCs attempt to communicate with patients, living donors, and families.

\section{One CTC described:}

The coordinator seems to be important based on being able to say, in such a kind of weak tone, that it is easy for the patient to talk to, easy to tell, or easy to say. (I)

\subsection{3 [Communication with a flow in response to the other person's reaction]}

The category of [communication with a flow in response to the other person's reaction] consists of two concepts: con- versation that immediately recognizes and responds to the other's reaction and an effort to offer explanations that correspond to the reaction of patients and families.

[Communication with a flow in response to the other person's reaction] is a communication skill that is useful in interviews and explaining transplantation. Conversation that immediately recognizes and responds to the other's reaction refers to changing the conversation content without breaking the flow of conversation in the response to the other's reaction, in which effective communication is attempted. CTCs make an effort to offer explanations that correspond to the reaction of patients and families upon assessing the other's reaction and conditions, and provide information for the other to form a correct understanding.

One CTC described:

As for the inquiry, the coordinator listens to the opponent for various things, so that if the story flies around here, those who ask questions also are very frustrating. When asking a flowing question, I think that the other person provides necessary questions and answers. (C)

\subsection{4 [Engaging so that patients and families do not have regrets after a transplantation]}

The category of [engaging so that patients and families do not have regrets after a transplantation] consists of five concepts: transplant selection support toward a family consensus, involvement toward a decision upon understanding the possible difficult outcome, promote unity of the family before the surgery, involvement with a long-term perspective, and determine support capability based on the relationship and wishes of the family.

CTCs understand that the post-transplantation prognosis depends on the self-management of patients, and family support is essential. Thus, they value [engaging so that patients and families do not have regrets after a transplantation], providing transplant decision support toward a family consensus, and determining support ability based on the relationship and wishes of the family; they aim to reach a transplant decision with a family consensus by building a family support system. If the family does not appear unified, CTCs will attempt to promote unity of the family before the surgery.

\section{One CTC commented:}

I think that transplantation is important for us to be aware of the treatment that survives with the family, not only with patients and donors, from the beginning. (B)

In addition, they become involvement to encourage mak- 
ing a choice based on an understanding the possibility of a poor outcome (e.g., complications and graft failure after transplantation), to provide decision-making support without post-transplantation regret for the patients and families. Upon assessing the lifestyle of patients and families, they became involved with a long-term perspective.

One CTC described:

I think that preparation and determination before transplantation are important. If it is solid, how can you spend your life when the kidneys become useless, register the next second transplant, choose home dialysis, live like there are no complications? I think I can choose to go. (F)

\subsection{5 [Provide support for the self-determination process and advocate the decision]}

The category of [provide support for the self-determination process and advocate the decision] consists of three concepts: promote self-decision with awareness, support the patient's decision and advocate, and support the process of accepting the disease and moving toward transplantation.

To [provide support for the self-determination process and advocate the decision], CTCs consider the patients' opinions and lifestyle to prevent patients and families from regretting the procedure, regardless of the transplantation results, and promote self-decision with awareness. Even if the patient's decision is not to go through with the transplantation, CTCs support the patient's decision and advocate by practicing decision-making support. In addition, CTCs are involved so that patients can support the process of accepting the disease and moving toward transplantation.

One CTC described:

I do not think that transplantation alone is a good treatment. I think that I do not have to do it if I decide that the person does not do it, and that if I choose it properly, I will lend $120 \%$ to the decision. (F)

\subsection{6 [Autonomous judgment and timely response while assessing patient conditions]}

The category of [autonomous judgment and timely response while assessing patient conditions] consists of four concepts: voluntary information collection toward timely medical intervention, response to transplant suitability determination with full use of medical knowledge, and timely presentation of the patient's problems, and noticing changes in patients' conditions.
[Autonomous judgment and timely response while assessing patient conditions] is a useful practice throughout the transplantation process. At the stage when a patient is introduced to transplantation, test data are gathered to determine transplantation suitability, and CTCs create a response to determination transplant suitability with the full use of medical knowledge. Instead, of leaving patient conditions to physicians, CTCs collect voluntary information with the aim of timely medical intervention for appropriate medical intervention.

One CTC described:

I will go and collect the patient's medical information myself without waiting for the doctor's instructions. ( $\mathrm{J}$ )

In addition, upon fully understanding the transplantation patient in their care, CTCs make a timely presentation of the patient's problems, and support an effective transplantation team conference. During outpatient treatment, CTCs check patient data and makes detailed observations of conditions within a short period of time, and upon noticing changes in the patient's conditions, they facilitate the early discovery of abnormalities.

One CTC commented:

First, I select the agenda for the conference. I cannot select the agenda unless I have grasped the whole picture of the hospitalized patient. (C)

\subsection{7 [Coordination in response to a change in conditions]}

The category of [coordination in response to change in conditions] consists of two concepts: providing appropriate information for a prompt adjustment and correction of sudden problems encountered during the transplantation process.

[Coordination in response to change in conditions] was practiced as an adjuster. When examining a living donor during an emergency or performing a preoperational test on a recipient immediately before deceased donor transplantation, CTCs provide appropriate information for a prompt adjustment. Because transplantation is associated with many sudden occurrences (e.g., deceased donor transplantation), CTCs make corrections for sudden problems encountered during the transplantation process, allowing for safe transplantation surgeries through smooth adjustments and responses.

One CTC described:

If there is a little problem or distortion, I think that I must have the ability to correct it each time. I will fix trouble with the doctor, trouble with the nurse, trouble in the examination room, trouble in the drug department, etc. (B)

ISSN 1925-4040 E-ISSN 1925-4059 


\subsection{8 [Collaborative care that is aware of team medical care]}

The category of [collaborative care that is aware of team medical care] consists of four concepts: conscious information sharing with other occupations, cooperation upon valuing care by ward nursing staff, heartfelt response to motivate those involved with organ donation, and an attempt to unify treatment policy among medical staff.

CTCs not only become involved with patients and their families but also provide [collaborative care that is aware of team medical care]. Although patients are hospitalized, through cooperation upon valuing care by ward nursing staff and conscious information sharing with other occupations, CTCs aim to provide the best care for patients and families. In addition, CTCs attempt to unify treatment policies among medical staff in such a way that there is no discrepancy in explanation contents of team members to the patient, and a relationship of trust is established.

\section{One CTC described:}

While consulting with a doctor, we decided on a treatment policy of "Let us explain in such a way." After these discussions, the doctors and/or I talk to the patient because the patient will be confused about what to do if the medical staff says different things. (D)

In addition, they attempt to unify treatment policies among medical staff to maximize team medical care. Because deceased donor organ transplantation cannot be completed by the team medical care within a hospital, CTCs practice with a heartfelt response to motivate those involved with organ donation (e.g., showing appreciation from the recipient to those involved with organ donation).

One CTC described:

Because Donor Coordinators in the Japanese organ transplant network cannot see the actual condition of the recipient getting better, they do not get a lot of motivation. When I informed them of the recipients' gratitude feelings, they were very pleased. In the past, I was doing business condition reporting on the recipients, but I think that it is important to put a heart to it, even though it takes time. (C)

\subsection{9 [Assertive communication with physicians]}

The category of [assertive communication with physicians] consists of three concepts: attitude in which one speaks up to physicians, strength to express one's opinion to physicians without hesitation, and identify the physician's social position and ability.

Published by Sciedu Press
[Assertive communication with physicians] is also shown as an autonomous practice of CTCs. If it is medically necessary, CTCs take an attitude in which one speaks up to physicians as their equal. They have the strength to express one's opinion to physicians without hesitation and perform necessary adjustments using assertive communication. By identifying the physician's social position and ability depending on the topic, CTCs practice consultation with physicians in a manner which derives an appropriate solution promptly.

\section{One CTC commented:}

I need to tell doctors what I should say. I think that it is useful to tell information properly and to tell your opinion properly. (C)

\subsubsection{0 [Mediate to build trust between patients/families and medical staff]}

The category of [mediate to build trust between patients/families and medical staff] consists of four concepts: develop the patient's confidence with medical care, mediate the communication between patients/families and medical staff, explanatory support for families to be convinced, and supplementation and explanation of a physician's explanation.

In [mediate to build trust between patients/families and medical staff], CTCs display trust of the physician in front of patients to develop the patient's confidence with medical care to form a relationship in which patients can receive medical care at ease. CTCs notice problems between the patients/families and physicians, and mediate communication between patients/families and the medical staff to maintain a good relationship between the two parties. In providing supplementation and clarification of the physician's explanation, CTCs encourage physicians to provide additional explanations and present supplementary information. CTCs pay attention not to cause confusion and that patients and families understand the explanations provided by physicians. For the families of a patient in poor condition, CTCs explain the situation, including difficult aspects, and provide opportunities to have a physician explain the condition if necessary. In this manner, they provide explanatory support for families to be convinced.

One CTC described:

Since the patient's family also has a relationship of trust with the coordinator, this can be a source of complaints to other medical personnel from patients and families. Patients and families sometimes conflict with nurses, so in such cases we will keep on getting on with each other and let it go smoothly. (K) 


\subsubsection{1 [Long-term care as a permanent primary]}

The category of [long-term care as a permanent primary] consists of five concepts: continued involvement toward a solution to difficulties faced in daily life, long-term care as a permanent primary, follow-up of the patient over a long period, a deep understanding the patients and families, and consultation suited to actual lifestyle.

CTCs are involved with patients over a long period of time from when they desire the transplantation to after the transplantation, and while closely following up with lifestyle and conditions that change, they practice [long-term care as a permanent primary]. First, by deeply understanding the patients and families, CTCs develop trust with patients and families, and continue to be involved toward a solution to difficulties faced in daily life. This is so that patients undergoing immunosuppressive therapy, to whom CTCs have become someone that the patients and families can rely on and consult with can enjoy life after the transplantation. They also provide consultations suited to their actual lifestyle for nonmedical aspects.

\section{One CTC described:}

Even trivial things such as whether it is life after leaving hospital, job searching, going on to school, traveling or something that is trivial, so that life as a role to receive such consultation I think that I would like to stay close. (J)

Through following up with the patient over a long period, CTCs remain involved while patients understand possible future complications and practice continued care.

\section{One CTC said:}

Whether you see that person in the long run or on the spot, the vision is different. Because the coordinator has been involved since the very beginning, I aim to lead things that I am doing now; for example, keeping internal organs properly will allow a long life after 30 years. (G)

\subsubsection{2 [Promoting self-monitoring by grasping the timing of guidance]}

The category of [promoting self-monitoring by grasping the timing of guidance] consists of four concepts: occasionally taking on a role to scold, capture the timing of guidance, promote self-monitoring, and guidance to start from easily achievable goals.

During the post-transplantation education of patients, CTCs [promoting self-monitoring by grasping the timing of guidance]. As the patient continues with self-monitoring, CTCs practice the promotion of self-monitoring that allows one review his or her own mistake and make corrections before discharge. After discharge, CTCs continue to be involved by providing guidance to start from easily achievable goals. Regarding long-term outcomes, by capturing the timing of guidance during changes in data and milestones (e.g., routine hospitalization), CTCs provide guidance whereby patients can review their lifestyle.

One CTC described:

In the period from the previous hospitalization to the regular examination hospitalization at this time, I will ask you to look back on "how can I forgot to take the medicine." (I)

By occasionally taking on a role to scold, CTCs encourage patients to continue with appropriate self-management.

One CTC described:

The medical staff is not to scold the patient? I think that we have the role of encouraging reprimand by saying that "you should be responsible for choosing a transplant without continuing alternative medicine for a long time." It is said that scolding, is of course, important. (D)

\subsubsection{3 [Stepping forward to solve problems]}

The category of [stepping forward to solve problems] consists of three concepts: more than makeshift problem solving, an attitude of reaching out and solving a problem together, and additional burden is acceptable as long as it helps the patients.

CTCs always have an attitude of [stepping forward to solve problems]. CTCs have an attitude of reaching out and solving a problem together toward patients, and find that an additional burden is acceptable as long as it helps the patients. If necessary, CTCs go beyond business hours, and provide more than makeshift problem solving for even the smallest issue a patient may have.

CTCs described:

I think the patient/family recognizes a coordinator who understands themselves and who can do something to solve problems or to think it through together. (A)

If a donor candidate or patient seems to want to talk about something, we hear the story of that person even when working time would exceed by 1 or 2 hours, thinking it was a waste of time; however, I think that nobody is useless. (B) 


\subsubsection{4 [Evaluate their own ability appropriately and have} humble attitude]

The category of [evaluate their own ability appropriately and have humble attitude] consists of four concepts: an attitude of continuous reflection on their own practice each day, expand the range of practice through self-improvement based on experience, awareness of a category for which one can be responsible, and an attitude of straight forward questions and good listening skills.

CTCs [evaluate their own ability appropriately and have humble attitude] to practice their jobs appropriately and better. In consultation with patients, it was acknowledged that making judgments and responding with medical knowledge upon gaining awareness of a category for which one can be responsible, and responding with an attitude of straight forward questions and good listening skills toward cooperation among team members is important.

One CTC described:

Because I am left by the doctor to say the whole thing, it is impossible to judge medical issues with the knowledge of nursing, so I judge whether it is necessary to contact a doctor. But the more it is done, the more it will gradually expand its scope. At first, I had confirmed all medicines with the doctor, but if I had more experience, I would not answer. As such, the scope of my judgment spreads little by little, but the new CTC is confused as to what to do when such a thing being asked by the patient. (K)

CTCs have an attitude of continuous reflection on their own practice each day so that patients and families can receive even better care, and are aware of expanding the range of practice through self-improvement based on experiences as CTCs.

One CTC described:

When the patient did not make it in time for the operation, I thought that it would be better if I could hurry the examination for operation at that time. After all, if the patient dies, I sometimes regret it because I should have told the family a little more at that time or explained that the risk is somewhat higher. $(\mathrm{J})$

\section{Discussion}

\subsection{Practice in decision-making support during trans- plantation selection}

Organ transplantation is a treatment used to save patient lives or improve physical conditions. Therefore, the ex- pectations of both patients, as well as living donor candidates and families' regarding transplantation treatment is high. However, the 1-year survival rate of liver transplant patients in Japan is $81 \%$, and it is possible that patients could die in early post-transplantation period. ${ }^{[3]}$ The prognosis of transplantation is not limited to those complications directly linked to only that surgery. Treatment compliance of post-transplantation patients also impacts the prognosis of patients; thus, post-transplantation patients must strictly adhere to management of immunosuppressants and cautions in their daily lives. However, Tanimizu et al. (2014) reported that in ethical settings in which the degree of concern raised by nurses is high, the "nurses questioned patients receiving transplantation when they see recipients who are unable to adhere to self-management," "in deciding transplantation treatment, they felt that recipients might have decided on transplantation without acknowledging the importance of post-transplantation self-management"; [23] thus, insufficient self-management by patients leads to an ethical dilemma. The practice of decision-making support presented in our study is very important for CTCs. CTCs' decision support began with [Observation to understand the real intention] when CTCs first touched base with the patient and their family. CTCs made careful observations to examine readiness for transplantation to determine if the patients, families, and living donor candidates actively desire transplantation, or if they are hesitating. In Japan, compared to deceased donor organ transplantation, living donor organ transplantation is much more common; ${ }^{[3,4]}$ thus, there were characteristics for living donor organ transplantation (e.g., understanding hesitations living donor candidates have about donation and capturing problems based on the facial expressions and words of living donor candidates and families). Previous studies have shown that the intention to donate by living donors in living donor transplantation is impacted by family dynamics. ${ }^{[24,25]}$ In particular, in cases of sibling living liver donors, sibling donors who are younger than the recipients tend to hesitate in making the donation, and it is often necessary to present the significance of the donation in addition to saving the recipients' lives to convince them to donate. ${ }^{[26]}$ This demonstrates that the relationship between patients and living donor candidates creates a strong intention to donate or conflict associated with donating. The refusal to donate by living donor candidates is an important decision that directly affects the patients' lives; thus, the decision making of living donor candidates is extremely delicate.

Therefore, the category of [Observation to understand actual intention] described the CTC's practice to assess the expectation of transplantation at the first interview with the patient and family. Also, CTCs caught inherent problems associ- 
ated with patients and families during a consultation through [conscious communication toward compromise], despite like first time to patient and family. As such, CTCs' examination of complex feelings and true intensions of families, as well as providing support to solve such issues are essential and require nursing skill.

Most characteristic in the decision-making support provided by Japanese CTCs was [engaging so that patients and families do not have regrets after transplantation]. Involvement toward a decision upon understanding the possible difficult outcomes showed that CTCs step in to explain the negative aspects and possibility of a poor outcome to patients and their families. This is an involvement to prepare patients for the chance of a poor outcome, and is a form of anticipatory grief care. Transplant decision support toward a family consensus upon assessing the family dynamics is a form of comprehensive care for patients, living donor candidates, and families. It is important to choose transplantation medical care with the autonomous opinion of the patients and living donor candidates. However, if the families' intentions are not in agreement, the relationship of the family could be destroyed. According to a prior report, liver donors may be blamed by families and relatives if the patient dies after transplantation (e.g., "why did you do the surgery? If you had not decided on the operation, he/she could be have lived longer, or he/she would not have to suffer." ). ${ }^{[27]}$ Japanese culture tends to suppress self-assertion, and issues are often considered as a family unit. ${ }^{[28]}$ Thus, CTCs consider it important to understand the patients' family background, relationships, and feelings of family members. As such, the CTCs' need for deep insight to understand complex feelings and true intensions of families as well as for providing support to solve such issues is essential and requires nursing skill.

Categories of involvement with long-term perspectives and determine the support ability based on the relationship and wishes of the family mean assessing drug administration compliance, including the patient's self-management and longterm family support system. The transplantation surgery is not the goal of treatment, but the beginning of transplantation medical care. CTCs provide information support regarding the future of post-transplantation life for the patient and family and support them so that they can imagine the actual life after transplantation. This is necessary for decision-making for transplantation.

This study showed that CTCs provide comprehensive support for decision-making to recipients and living donors. In the US, there are CTCs who are in charge of only living donors, ${ }^{[29]}$ and play the role of defending living donor candidates. $^{[30]}$ In Japan, because the number of CTCs is small, ${ }^{[8]}$ CTCs are in charge of both the recipients and living donors. Being in charge of subjects with potentially opposing values can lead to an ethical dilemma of who to prioritize. However, Japanese CTCs practice decision-making support upon acknowledging that an approach to the family based on Japanese culture, which values the consensus of the family is important. As such, CTCs take on the ethical dilemma unique to transplantation, whereas supporting decision-making to avoid post-transplantation regret for patients and families. This is characteristic in the decision-making support provided by Japanese CTCs, and the CTC was an ethics coordinator, so we considered this expert practice.

\subsection{Practice in coordination during the transplantation process}

A category of [autonomous judgment and timely response while assessing patient conditions] showed CTC effort to effectively examine patients' problems is made to understand the medical conditions of waitlisted and transplant patients, and determine the priority of each problem. In addition, with response to determining transplant suitability with full use of medical knowledge, if a patient who may be suitable for transplantation is introduced, CTCs lead information gathering to determine the indication for transplantation. In the US, the initial evaluation of patients before transplantation is the responsibility of CTCs who are advanced practical nurses (APNs). ${ }^{[19]}$ As it has been reported that Japanese nurse specialists practice nursing that fuse cure and care (e.g., gathering information based on medical knowledge and leading to a diagnosis by physicians ${ }^{[31]}$ ), an accurate physical assessment is an advanced nursing practice. As such, the practice of CTCs who were subjects of this study is similar to the practice level of APNs. However, because there is no nurse specialist system in the field of transplantation nursing in Japan, CTCs gain their skills through self-learning upon generalist nurse experience, and provide advance practice. The other hand, even in the US, CTCs practice in the range dictated by the condition of each facility, and depending on the situation, it was reported the boundary with APNs becomes unclear. ${ }^{[20]}$ Therefore, it is a necessary future challenge to construct the educational system for CTCs who practice expertise as [autonomous judgment and timely response while assessing patient conditions] in the Japanese nursing system.

[Assertive communication with physicians] such as an attitude in which one speaks up to physicians and the strength to express one's opinion to physicians without hesitation were necessary communication methods to achieve an autonomous decision and response by CTCs. This is consistent with the previous study which the communication style of CTCs is more assertive and flexible than that of transplanta- 
tion staff nurses, and they function more independently than transplantation staff nurses regarding the management relationship with physicians, patients, and other medical staff. ${ }^{[32]}$ Depending on the content of the consultation, the concept of identifying the physician's social position and ability is an approach toward making an appropriate and prompt solution to a problem upon understanding the characteristics of physicians involved with CTCs. Assertive communication of CTCs not only expresses their opinions to physicians but also attempts at effective communication that identifies the person they are counseling to derive appropriate judgment. It was shown that [Assertive communication with physicians] is a skill supporting autonomous practice as CTC.

CTCs play a role of cooperating with other medical experts to provide the care necessary for patients. ${ }^{[2]}$ A category of [Collaborative care that is aware of team medical care] descibed that CTCs had promoted information sharing and discussion among medical team members, and had adjusted conditions to maximize the roles and abilities of each team member. This was not only an adjustment for multi-occupation collaborations but also to respect and nurture the competency of many occupations. To [mediate to build trust between patients/families and medical staff], CTCs were involved to avoid a sense of distrust in patients because of insufficient communication between patients/families and medical staff. Consistent communication and team building functions are important for the safety and well-being of patients. ${ }^{[33]}$ CTCs did not only develop a relationship between patients and families but also connect the relationship between patients/families and medical staff to provide medical care that patients and family consider safe. Therefore, the CTC has demonstrated expertise practice of coordination for the multidisciplinary team to ensure patient safety and quality of care.

CTCs monitored the transplantation process in individual cases, and practice [coordination in response to changes in conditions]. In cadaver organ transplantation procedure, in addition to regular work, CTC have to do the adjustment of human resources, communication within the hospital, and surgical preparation are necessary for the surgery, as quickly as possible. CTCs noticed small problems under these conditions and respond. Benner et al stated that nurses not only provide direct care for the urgent physiological needs of patients who require treatment in an emergency but also prepare, organize, and adjust for various aspects surrounding the situation. ${ }^{[32]}$ In particularly critical situations, expert nurses must look past the situation before them, and make an adjustment to the overall situation. CTCs grasped the overall situation of the transplantation process, and adjusted for the detailed problems in the transplantation process. There-

Published by Sciedu Press fore, [coordination in response to changes in conditions] was shown to be an expertise practice of CTCs using crisis management abilities.

\subsection{Practice in long-term continuous care}

Preventing post-transplantation noncompliance and patient education are an important role of CTCs. ${ }^{[29]}$ The practice of [promoting self-monitoring by grasping the timing of guidance] in daily life guidance after the transplantation was for CTCs to become involved to promote patients' selfmonitoring, so that patients can change their behavior by noting changes in their bodies and data. Benner et al. (2005) stated that it is necessary for physicians to teach patients to "read" the reaction of their own body as a prerequisite of self-management. ${ }^{[33]}$ As same, CTC's made patients aware of their data and changes in physical conditions that are effective for a patient's self-monitoring skills. CTCs also had planned the timing of instruction, which is an effective form of involvement for patients to continue with their selfmonitoring. In addition, CTCs provided patient education with a long-term perspective for 10 and 20 years in the future, even if there is no abnormal data at the present and there are no problems with the patient's physical condition, which was a characteristic practice of CTCs who are involved with patients over a long period after transplantation. This continuous guidance by CTCs was cultivated from experience knowledge of physical problems of transplant patients that occurred in the long-term course. Therefore, we demonstrated that the CTC's practice is based on clinical foresight to predict and prevent potential problems.

The function of CTCs in outpatient care is triage and medical intervention in the outpatient clinic. ${ }^{[6]}$ The Japanese CTC's practice of [Long-term care as a permanent primary] involves their evaluation not only of medical issues but also the problem of daily life that patients face after discharge. In this practice, it is important to maintain a relationship with the patients and their families so that they will consult the CTC without hesitation. We suggest that this practice of CTCs was based on the recognition that even a problem of daily life unrelated to a medical issue may affect the outcome and long-term prognosis of the patient. This is a unique practice of CTCs in Japan to improve patient quality of life.

\subsection{Limitations of this study and future issues}

Although this study described the expertise practice of Japanese nurse CTCs, it is possible that these specialized practices have been accumulated from each nursing experience and not based on evidence. However, the expertise practice of CTC clarified in this research is useful for to improve and assess the quality of care of CTC. In the fu- 
ture, an educational program is necessary to nurture CTCs conducting expertise nursing practice. The results from this study suggest that it is imperative to research Japanese CTCs' advanced nursing education program construction based on their expertise practice.

\section{Conclusions}

CTC in Japan demonstrates expertise practice with decision support during transplant selection, coordination during the transplant process, and long-term continuing care. The expertise practice of Japanese CTCs was developed as an advanced nursing practice similar to that in Western countries because of accumulating nursing practice as a generalist from experience as CTCs. In addition, this study highlighted that CTCs in Japan had been conducting autonomous practice as a nurse and providing comprehensive care including patients, family members, and living donors. Our results clarified the expertise practices of CTCs experienced in all organ transplants. We suggest that these results can be used to improve and assess the quality of care given by Japanese CTCs in the transplant process.

\section{ACKNOWLEDGements}

We are grateful for the dedication and shared insights of all our participants and co-investigators. We thank Crimson Interactive Pvt. Ltd. (Ulatus)-www.ulatus.jp for their assistance in manuscript translation and editing.

\section{CONFlicts of InTEREST Disclosure}

The authors declare that there is no conflict of interest.

\section{REFERENCES}

[1] Organ Procurement and Transplantation Network [cited 2017 December 10]. Available from: https://optn.transplant.hr sa.gov/news/us-organ-transplants-and-deceased-don ors-set-new-records-in-2016/

[2] Japan Organ Transplant Network [cited 2017 December 10]. Available from: https://www.jotnw.or.jp/datafile/offer_bra in.html [in Japanese]

[3] The Japanese Liver Transplantation Society: Liver Transplantation in Japan-Registry by the Japanese Liver Transplantation Society. Japanese Journal of Transplantation [Nihon Ishoku Gakkai]. 2017; 52: 134-147. http://doi.org/10.11386/jst.52.2-3_134 [in Japanese]

[4] The Japan Society for Transplantation, Japanese Society for Clinical Renal Transplantation: Annual progress report from the Japanese Renal Transplant Registry: number of renal transplantation in 2015 and follow-up survey. Japanese Journal of Transplantation [Nihon Ishoku Gakkai]. 2017; 52: 113-133. http://doi.org/10.11386 /jst.52.2-3_113 [in Japanese]

[5] Donaldson TA. The role of the transplant coordinator, Transplantation Nursing Secrets. Hanley \& Belfus, Inc. 2003. 17-26 p.

[6] Ohler L, Shafer T. Advanced practice: implication for clinical and procurement transplant coordinators. Prog transplant. 2006; 16: 103-104. https://doi.org/10.1177/1526924806016002 01[PubMed:16789697]

[7] Hagiwara K, Iyama N, Kubota K, et al. Survey of Japanese recipient transplant coordinator. Japanese Journal of Transplantation [Nihon Ishoku Gakkai]. 2000; 35: 151. [in Japanese]

[8] Nakajima S, Hagiwara K, Soeda E, et al. Survey of Japanese recipient transplant coordinator-Japanese Transplant Coordinator Organization. Journal of Japan Academy Transplantation and Regeneration Nursing. 2009; 5: 28. [in Japanese]

[9] American Nurses Society: International Transplant Nurses Society. Transplant Nursing Scope and Standards of Practice. 2nd ed. Sliver Spling. 2016.

[10] Hagiwara K. Mission and roles of certified transplant recipient coordinators. Organ Biology [Nihon Zoki Hozon Seibutsu Igakkai]. 2012; 20: 5-11. [in Japanese] http://doi.org/10.11378/organbio. 20.5
[11] Umeki E. Real Situations of recipient transplant coordinator/renal transplantation. Japanese Journal of Transplantation [Nihon Ishoku Gakkai]. 2005; 40: 33-40. [in Japanese]

[12] Nojiri K, et al. Real situation of recipient transplant coordinator/liver transplantation. Japanese Journal of Transplantation [Nihon Ishoku Gakkai]. 2005; 40: 11-16. [in Japanese]

[13] Saito M, et al. Real situation of recipient transplant coordinator/lung transplantation. Japanese Journal of Transplantation [Nihon Ishoku Gakkai]. 2005; 40: 25-32. [in Japanese]

[14] Hagiwara K: Decision making by families in living donor transplantation. Family Nursing Vol. Nihon Kangkyoukai Syuppankai, Tokyo. 2003. 104-111 p. [in Japanese]

[15] Hagiwara K. Support to living donor candidates before transplantation/necessity of support to living donor candidates in living organ transplantation. Transplantation Now [Konnichi no ishoku]. 2007; 20: 364-366. [in Japanese]

[16] Hagiwara K. Support for Living Renal Transplantation Decision. Journal of Osaka Society of Dialysis Therapy. 2008; 26: 79-83. [in Japanese]

[17] Sijiki Y. A Study on Role and Function of Nurse Transplant Coordinator in Organ Transplantation Medical Care-Focus on Living Partial Liver Transplantation, Grant Number 16390627 2004-2006 Grantin-Aid for Scientific Research (B) (2) Study result report. March 2007. [in Japanese]

[18] Shimizu J. A Study on Development and Evaluation of Clinical Transplant Coordinator Nurse Education Program, A Study on Development and Evaluation of Organ Transplant Coordinator Nurse Education Program, (2007 redo-Wakate-001), ministry of Health, Labour and Welfare, Grant-in-Aid for Scientific Research Grant Regeneration Medical Research Project, 1998 Comprehensive/Shared Research Report. March 1999. [in Japanese]

[19] Hoy H, Alexander S, Payne J, et al. The role of advanced practice nurses in transplant center staffing. Prog Transplant. 2011; 21: 294-298. https://doi.org/10.1177/1526924811021004 07 [PubMed:22548990]

[20] Coleman B, Blumenthal N, Currey J, et al. Adult cardiothoracic transplant nursing: An ISHLT consensus document on the current adult nursing practice in heart and lung transplantation. J Heart Lung Translpant. 2015; 34: 139-148. 
[21] Kinoshita Y. Practicing Grounded Theory Approach [Invitation to Qualitative Research], Koubundou, Tokyo. 2003. [in Japanese]

[22] Kinoshita Y. Live Lecture M-GTA-Practical Qualitative Research Method, The Whole Modified Grounded Theory Approach (first version), Koubundou, Tokyo. 2007. [in Japanese]

[23] Tanimizu N, Imanishi T, Shuda A, et al. The Existence of Experience and Frequency and Severity of Related Concerns of Ethical Issues in Nursing Practice Encountered in Organ Transplantation. Transplant Proc. 2014; 46: 1029-1031.

[24] de Villa VH, Lo CM, Chen CL. Ethics and rationale of living-donor liver transplantation in Asia. Transplantation. 2003; 75: S2-S5.

[25] Fujita M, Slingsby BT, Akabayashi A. Three patterns of voluntary consent in the case of adult-to-adult living related liver transplantation in Japan. Transplant Proc. 2004; 36: 1425-1428. http: //dx.doi.org/10.1016/j.transproceed.2004.04.088

[26] Hagiwara K, Seto N, Shimizu Y, and others. Factors affecting the decision-making process of live donors who have their own family and donate their liver to a sibling, Journal of Japan Academy of Nursing Science [Nihon Kango Kagakkai shi]. 2013;33:66-73. http://doi.org/10.5630/jans.33.3_66

[27] Japanese Liver Transplantation Society, Donor Report on Living Liver Transplantation, March 2005, http://jlts.umin.ac .jp/images/donor_survey_summary.pdf, 2017/8/13 [in Japanese]

[28] Komatsu N. Door of Medical Ethics—About Life and Death—, First Edition First Press, Hokuju, Tokyo 2005. 38-50 p. [in Japanese]

[29] Sites AK, Freeman JR, Harper MR, et al. A multidisciplinary program to educate and advocate for living donors. Prog Transplant. 2008; 18(4): 103-104. https://doi.org/10.1177/1526924808 01800411

[30] Gwen E, McNatt GE. Nursing and transplant coordination: a call for clarity. Prog Transplant. 2008; 18: 208-215.

[31] Arakawa Y, Inoue T. Analysis of Internal Structure of Nursing Practice that Integrates Cure and Care Toward Development of Nursing Care (Integrating Care and Cure in Nursing Practice in Japan). Journal of Japan Academy of Nursing Science [Nihon Kango Kagakkai shi]. 2015; 35: 72-81. https://doi.org/10.5630/jans. 35.72152

[32] Pennington M, Lancaster W. Paris: A comparison of role expectations and communication styles between transplant coordinators and transplant staff nurses. Journal of Transplant Cordinator. 1998; 8: 119-124.

[33] Benner P, Hooper-Kyriakidis PL, Stannard D. Clinical Wisdom in Nursing Care-Thinking while Acting (Clinical Wisdam and Intervention in Clinical care). Igaku-Shoin, Tokyo. First Edition First Press; 2005. 